\title{
Systematic review of paediatric track and trigger tools for identifying clinical deterioration of children in hospital: their development, validation and effectiveness
}

Authors: Rob Trubey, Chao Huang, Fiona Lugg-Widger, Kerenza Hood, Davina Allen, Dawn Edwards, David Lacy, Amy Lloyd, Mala Mann, Brendan Mason, Alison Oliver, Damian Roland, Gerri Sefton, Richard Skone, Emma Thomas-Jones, Lyvonne Tume, Colin Powell

Aims

To assess how

(1) well validated existing paediatric track and trigger tools (PTTT) \&

(2) how effective paediatric early warning systems (with or without a PTTT)

are at reducing mortality and morbidity outcomes in hospitalised children.

\section{Methods}

A systematic search was carried out across several databases for studies reporting on the development, validation and effectiveness of PTTT, or broader early warning systems with or without a PTTT. Study selection, data extraction and quality assessment were conducted by two independent reviewers and disagreements resolved by discussion

\section{Most frequent components of published Paediatric Track \& Trigger Tools}

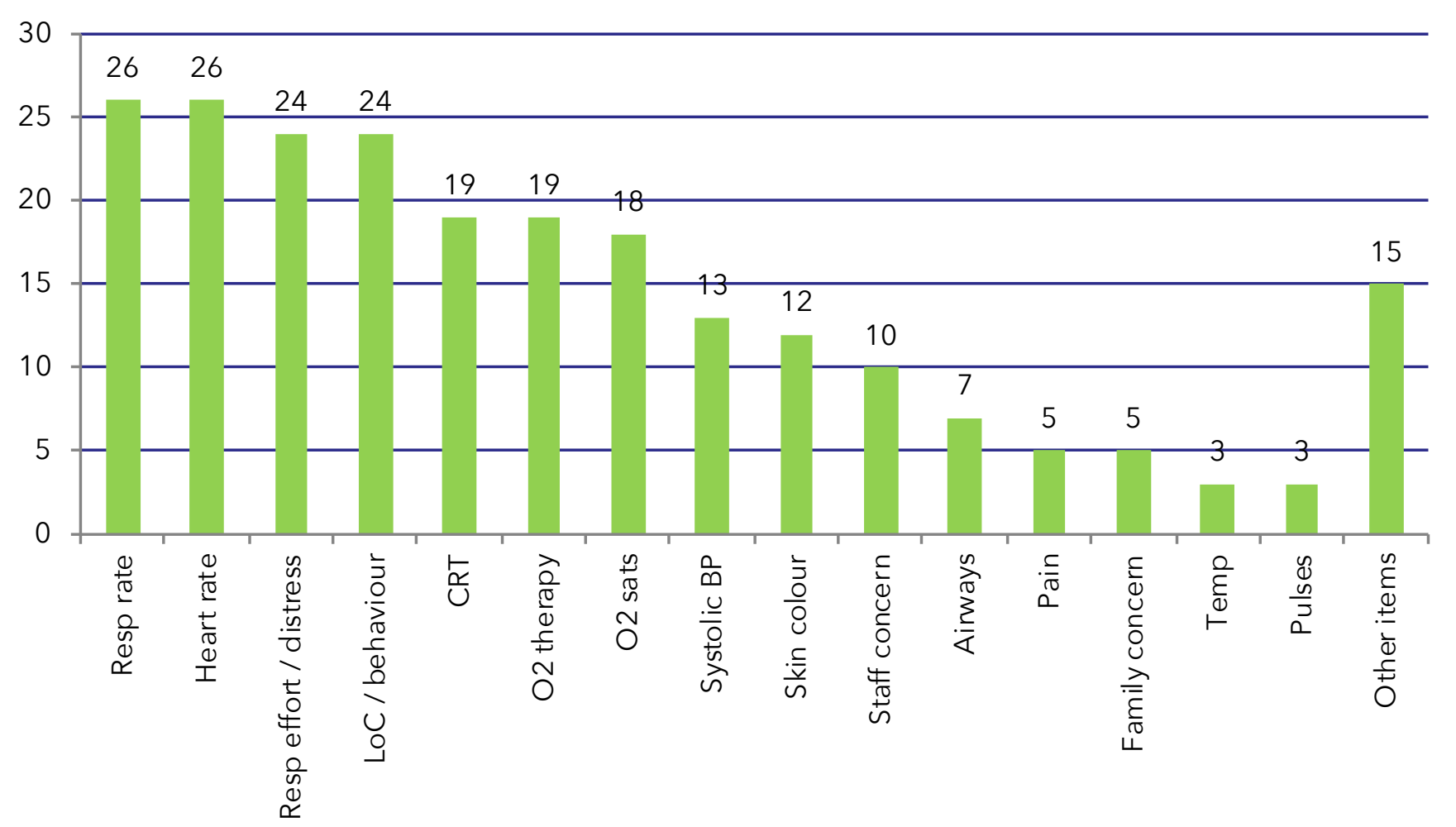

\section{Results}

36 validation studies and 30 effectiveness studies were included, predominantly from North America, Australia and the UK, and were largely single-site studies conducted in specialist centres.

Outcome measures varied considerably. Validation studies were largely retrospective, case-control studies: Positive predictive value was typically low, suggesting the potential for alarm fatigue.

No studies accounted for the co-occurrence of routine clinical intervention and the longitudinal nature of the predictors in evaluating the link between high PTTT scores and subsequent deterioration.

Single / multi-centre

Single-centre

Multi-centre

\section{Study population}

General in-patients

Specialist population

Unclear

Study design

Cohort

Case-control

Case / chart review

Pilot study

\section{Conclusion} supported by the evidence base.
$35 \quad 97.2$

Effectiveness studies $(\mathbf{n}=\mathbf{3 0})$

Country

United States

1860.0

United Kingdom

310.0

Canada

Australia

Other

Multiple

Unclear

Setting

Specialist / tertiary $\quad 29 \quad 96.7$

Non-specialist /

community

Unclear

Single / multi-centre

Single-centre

2893.3

Multi-centre

$2 \quad 6.7$

\section{Study population}

General in-patients

$20 \quad 66.6$

$23 \quad 63.9$

1130.6 Specialist population

Unclear

$5 \quad 16.7$

$\begin{array}{ll}5 & 16.7\end{array}$

Study design

$\begin{array}{lllll}7 & 19.4 & \text { Uncontrolled before-after } 26 \quad 86.7\end{array}$

$18 \quad 50.0 \quad$ Controlled before-after $\quad 1 \quad 3.3$

$\begin{array}{llll}10 & 27.8 & \text { Interrupted Time Series } \quad 2 & 6.7\end{array}$

\begin{tabular}{lllll}
1 & 2.8 & Cluster randomised trial & 1 & 3.3 \\
\hline
\end{tabular}

There are a number of fundamental methodological limitations in the PTTT literature, and a predominance of single-site studies carried out in specialist centres limits generalisability.

With limited evidence of effectiveness, we would argue that calls to make their use mandatory across all paediatric units are not 